\title{
Ocorrência de hiperóxia em unidade de terapia intensiva adulto de um hospital universitário do nordeste do Brasil
}

\section{Occurrence of hyperoxia in an adult intensive care unit of a university hospital in northeastern Brazil}

\author{
Lara Silva Aguiar ${ }^{1}$. Beatriz Amorim Beltrão². Natalia Linhares Ponte Aragão2 . Vitor Nogueira Araújo ${ }^{2}$. \\ Antônio Brazil Viana Júnior². João Gabriel Ávila Gomes ${ }^{1}$. Arnaldo Aires Peixoto Junior ${ }^{1,2}$. \\ 1 Universidade Federal do Ceará (UFC), Fortaleza, Ceará, Brasil. 2 Hospital Universitário Walter Cantídio (HUWC), Fortaleza, \\ Ceará, Brasil.
}

\section{RESUMO}

Objetivos: Hiperóxia é comum em pacientes críticos submetidos à ventilação mecânica nas Unidades de Terapia Intensiva (UTI) sem efeito benéfico e, em alguns casos, pode causar danos. Sua incidência varia de 16-50\% nesta população. Este trabalho tem como objetivo estudar a prevalência de hiperóxia em pacientes submetidos à ventilação mecânica na UTI do Hospital Universitário Walter Cantídio. Método: estudo observacional, retrospectivo, através da avaliação dos registros de pacientes internados em uma unidade de terapia intensiva adulto de um hospital universitário. Foram avaliados todos os pacientes adultos, submetidos à ventilação mecânica, internados consecutivamente, no período de março a setembro de 2017. Resultados: nesse estudo, foram analisados 102 pacientes internados na UTI submetidos à ventilação mecânica. A hiperóxia $\left(\mathrm{PaO}_{2}>120 \mathrm{mmHg}\right)$ foi encontrada em 23,5\% desses pacientes durante as primeiras 24 horas de admissão à UTI. Dos 102 pacientes analisados, 86 desses permaneceram na UTI após 72 horas da admissão e apenas $7 \%$ desses doentes continuavam com hiperóxia. Conclusão: os níveis de $\mathrm{PaO}_{2}$ nas primeiras 24 horas estavam frequentemente acima do recomendado. Esse fato justifica a necessidade de uma maior atenção à oferta adequada de oxigênio aos pacientes sob ventilação mecânica na prática diária.

Palavras-chave: Hiperóxia. Ventilação mecânica. Unidade de Terapia Intensiva. Pressão arterial de oxigênio.

\section{ABSTRACT}

Objectives: Hyperoxia is common in critically ill patients undergoing mechanical ventilation in Intensive Care Units (ICUs) without beneficial effect and in some cases may cause damage. Its incidence ranges from $16-50 \%$ in this population. This study aims to study the prevalence of hyperoxia in patients submitted to mechanical ventilation at the University Hospital Walter Cantídio ICU. Method: retrospective observational study through the evaluation of the records of patients hospitalized in an adult intensive care unit of a university hospital. All adult patients submitted to mechanical ventilation, hospitalized consecutively, were evaluated in the period from March to September 2017. Results: In this study, 102 patients admitted to the ICU were submitted to mechanical ventilation. Hyperoxia $\left(\mathrm{PaO}_{2}>120 \mathrm{mmHg}\right)$ was found in $23.5 \%$ of these patients during the first 24 hours of ICU admission. Of the 102 patients analyzed, 86 of these remained in the ICU after 72 hours of admission and only $7 \%$ of these patients continued with hyperoxia. Conclusion: $\mathrm{PaO}_{2}$ levels in the first 24 hours were often higher than recommended. This fact justifies the need for greater attention to the adequate supply of oxygen to patients under mechanical ventilation in daily practice.

Keywords: Hyperoxia. Mechanical ventilation. Intensive Care Units. Oxygen arterial pressure.

Autor correspondente: Lara Silva Aguiar, Rua Capitão Francisco Pedro, 1290, Rodolfo Teófilo, Fortaleza, Ceará. CEP: 60430-370. Telefone: +55 85 3366-8162. E-mail: lara_aguiar19@hotmail.com

Conflito de interesses: Não há qualquer conflito de interesses por parte de qualquer um dos autores.

Recebido em: 20 Fev 2018; Revisado em: 06 Out 2018; Aceito em: 06 Out 2018. 


\section{INTRODUÇÃO}

A hiperóxia em pacientes graves, submetidos à ventilação mecânica, é comum e sua incidência varia entre 16 a 50\% nesta população. ${ }^{1-5}$

Durante a permanência na unidade de terapia intensiva (UTI), a fração inspirada de oxigênio $\left(\mathrm{FiO}_{2}\right)$ aplicada geralmente excede as concentrações de oxigênio habitualmente encontradas no ar ambiente. Consequentemente, pacientes graves alcançam níveis elevados de pressão arterial de oxigênio $\left(\mathrm{PaO}_{2}\right)$ nas primeiras 24 horas de admissão na UTI. ${ }^{2}$

Em princípio, a hiperóxia pode compensar e prevenir hipóxia tecidual, promovendo a entrega de oxigênio para os órgãos afetados. ${ }^{6}$ Entretanto, muitos estudos sugerem que, provavelmente, a hiperóxia não é segura..$^{7-9}$

A hiperóxia pode resultar em um incremento na geração de radicais livres no sistema nervoso central, fígado e pulmão. ${ }^{10}$ Também está associado a vasoconstrição, diminuição do débito cardíaco e do transporte de oxigênio. ${ }^{7}$

Em um estudo experimental, a hiperóxia prolongada resultou em alterações histopatológicas semelhantes às observadas na Síndrome do Desconforto Respiratório Agudo. ${ }^{11}$ Em humanos saudáveis, a exposição a $100 \%$ de oxigênio foi associada a atelectasias, deficiência de depuração mucociliar, traqueobronquite, aumento da permeabilidade capilar e aumento dos neutrófilos alveolares. ${ }^{12}$

É relatado também um impacto negativo da hiperóxia na sobrevida em diferentes populações de pacientes, como os indivíduos submetidos à ressuscitação cardíaca, com acidente vascular cerebral isquêmico agudo e com infarto do miocárdio associado a elevação do segmento ST. ${ }^{7}$

Diante do exposto, a terapia com oxigênio produz um delicado equilíbrio entre benefício e dano, dependendo da dose, duração e doenças subjacentes. Em pacientes gravemente enfermos, os efeitos prejudiciais são acentuados e podem eventualmente prevalecer, considerando a duração prolongada do oxigênio suplementar e a susceptibilidade do paciente à inflamação e a presença de instabilidade cardiovascular. ${ }^{6}$

Neste cenário, é importante a suspeita deste diagnóstico nos pacientes submetidos à ventilação mecânica por parte de todos os profissionais das UTIs no intuito de evitar ou reduzir os efeitos deletérios induzidos pela presença de hiperóxia. Assim, desenhamos este estudo com o objetivo de identificar o percentual de ocorrência de hiperóxia dentre os pacientes ventilados mecanicamente na UTI adulto de um hospital universitário terciário do nordeste brasileiro.

\section{MÉTODOS}

Trata-se de estudo retrospectivo observacional, no qual foram analisados dados obtidos nos prontuários dos pacientes internados em uma UTI adulto do Hospital Universitário Walter Cantídio.

\section{População e variáveis}

Foram avaliados todos os pacientes adultos, internados consecutivamente, no período de março a setembro de 2017. Pacientes não submetidos à ventilação mecânica foram excluídos da análise. Foram colhidos dados demográficos, escores de gravidade Acute Physiology and Chronic Health Evaluation II (APACHE II) ${ }^{13}$ e Sequential Organ Failure Assessment (SOFA), ${ }^{14} \mathrm{PaO}_{2}$ na gasometria arterial colhida nas primeiras 24 horas após admissão, e $\mathrm{FiO}_{2}$ ofertada no momento da coleta da gasometria. Definimos hiperóxia como $\mathrm{PaO}_{2}$ maior ou igual a $120 \mathrm{mmHg} \cdot{ }^{10,15,16}$

\section{Análise estatística}

As variáveis categóricas foram descritas em frequência absoluta e relativa (percentual). Variáveis contínuas com distribuição normal foram expressas em média e desvio padrão, e as com distribuição não-normal em mediana e percentis. A comparação entre os dois subgrupos foi realizada pelo teste qui-quadrado de Pearson ou teste exato de Fisher para as variáveis dicotômicas; e pelo teste t de Student para variáveis contínuas. Para as variáveis que não seguiram uma distribuição normal foi utilizado o teste de Mann-Whitney. Os dados foram tabulados no software Excel 2007 para Windows ${ }^{\circledR}$ e as análises foram realizadas por meio do software SPSS Statistics $^{\circledR}$, versão 20.0.0.

\section{Aspectos éticos}

Este estudo faz parte de uma coorte retrospectiva, com coleta de dados registrados em prontuários, com dispensa do preenchimento de Termo de Consentimento Livre e Esclarecido. Foi aprovado pelo Comitê de Ética em Pesquisa da instituição (CAAE: 64529817.1.0000.5045), estando de acordo com a resolução 466/12 do Conselho Nacional de Saúde e a Declaração de Helsinque.

\section{RESULTADOS}

No período estudado, 135 pacientes foram admitidos na UTI. Dentre esses pacientes, 102 indivíduos atendiam aos critérios de inclusão. As características dos pacientes estão disponíveis na Tabela 1. A média da idade foi de $57 \pm 17$ anos, 53 indivíduos eram do sexo masculino (52,0\%), a média do escore APACHE II foi de $21 \pm 6$, e a do escore SOFA foi de $9 \pm 4$. As admissões clínicas predominaram em relação às cirúrgicas $(96,1$ e 3,9\%, respectivamente).

Dos 102 pacientes analisados, observou-se o percentual de ocorrência de hiperóxia $\left(\mathrm{PaO}_{2}>120 \mathrm{mmHg}\right)$ em 24 (23,5\%) dos indivíduos durante as primeiras 24 horas da admissão à UTI.

Não houve diferença com significância estatística entre os pacientes que estavam ou não em hiperóxia nas primeiras 24 horas quanto a idade, escores de gravidade APACHE II e SOFA e o tipo de admissão (clínica ou cirúrgica). Houve um 
predomínio do sexo feminino nos pacientes com hiperóxia nesse primeiro dia de UTI $(\mathrm{p}=0,011)$ (Tabela 1$)$.

Dentre os 102 pacientes incluídos no estudo, um total de 86 permaneceram por mais de 72 horas na UTI. Dentre esses
86 indivíduos, um total de 21 (24,0\%) indivíduos estavam em hiperóxia na admissão e $6(7,0 \%)$ permaneceram em hiperóxia na avaliação após 72 horas de internamento na unidade (Gráfico 01).

Tabela 1. Características da população sob ventilação mecânica com e sem hiperóxia à admissão na UTI.

\begin{tabular}{lcccc}
\hline & Total & Hiperóxia & Não hiperóxia & p \\
& $(\mathrm{N}=102)$ & $(\mathrm{N}=24)$ & $(\mathrm{N}=78)$ & $0,495^{\mathrm{b}}$ \\
\hline Idade (anos)* & $57 \pm 17$ & $60,46 \pm 15,33$ & $56,37 \pm 17,92$ & $0,011^{\mathrm{c}}$ \\
Sexo masculino** & $53(52,0)$ & $7(29,2)$ & $46(59,0)$ & $0,918^{\mathrm{b}}$ \\
Escore SOFA* & $9 \pm 4$ & $8,75 \pm 3,77$ & $8,9 \pm 4,34$ & $0,175^{\mathrm{a}}$ \\
Escore APACHE II* & $21 \pm 6$ & $22,79 \pm 6,32$ & $20,83 \pm 6,09$ & $0,570^{\mathrm{d}}$ \\
Tipo de admissão & & & $74(94,9)$ & $4(5,1)$ \\
$\quad$ Clínica & $98(96,1)$ & $24(100,0)$ & $0(0,0)$ & \\
Cirúrgica & $4(3,9)$ & &
\end{tabular}

Nota: * média \pm desvio padrão; ** N (\%). a: Teste t de Student; b: Teste de Mann-Whitney; c: Teste Qui-quadrado de Pearson; d: Teste Exato de Fisher.

Gráfico 1. Percentual de hiperóxia à admissão e após 72 horas em paciente sob ventilação mecânica na UTI (N=86).

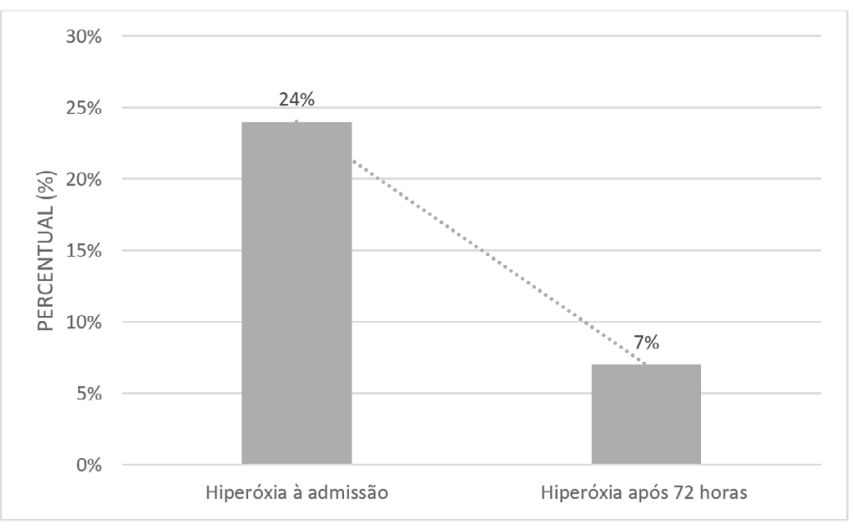

\section{DISCUSSÃO}

Nesse estudo observacional, retrospectivo, identificamos uma ocorrência elevada de hiperóxia, definida como $\mathrm{PaO}_{2}$ maior ou igual a $120 \mathrm{mmHg}$, no primeiro dia de internação na UTI (D1), com uma redução dessa, 72 horas após a admissão em pacientes submetidos à ventilação mecânica (D3). Essa ocorrência independeu da idade e da estimativa de gravidade através dos escores APACHE II e SOFA.

Em grandes estudos retrospectivos anteriores ao nosso, a incidência de hiperóxia nas primeiras 24 horas da admissão variou de 23 a 50\%, com $\mathrm{FiO}_{2}$ de 0,50 a 0,62. ${ }^{10,15}$ Em nossa população, a hiperóxia foi encontrada em $23,5 \%$ dos indivíduos no primeiro dia de ventilação mecânica, semelhante ao encontrado em um estudo de coorte retrospectivo realizado na Holanda, no qual foi evidenciado a presença de $\mathrm{PaO}_{2}>$ $120 \mathrm{mmHg}$ em $22 \%$ dos testes gasométricos, porém, na maioria dos casos, não ocorreu ajustes no ventilador. ${ }^{17}$

A hiperóxia é conhecida por ser prejudicial, mas quanto tempo ela pode ser tolerada e a relação dos níveis de $\mathrm{PaO}_{2}$ com os danos físicos ainda não foi esclarecida. ${ }^{16}$ A incidência aumentada de pneumonias associadas à ventilação, o prolongamento da permanência de ventilação mecânica e o maior tempo de internamento em UTI são observados. ${ }^{18,19}$ Por outro lado, uma redução nas infecções de sítio cirúrgico pelo uso de hiperóxia foi relatada por um grupo de estudo. ${ }^{20}$

Muitos estudos observacionais realizados em pacientes internados na UTI mostraram relação entre hiperóxia e mortalidade. Em um grande estudo retrospectivo na Holanda, houve associação em forma de U entre a pressão de oxigênio arterial alcançada $\left(\mathrm{PaO}_{2}\right)$ durante as primeiras 24 horas após a admissão com maior mortalidade em pacientes com $\mathrm{PaO}_{2}$ muito baixa ou alta. ${ }^{10} \mathrm{~A}$ mortalidade maior em pacientes com $\mathrm{PaO}_{2}$ muito baixa não é inesperada e possivelmente pode ser atribuído à seleção dos pacientes mais graves. No entanto, a mortalidade também foi maior nos pacientes com maiores valores de $\mathrm{PaO}_{2}$, sugerindo a possibilidade de toxicidade sistêmica do oxigênio.

Por outro lado, um estudo multicêntrico retrospectivo mostrou associação entre hipóxia e aumento da mortalidade hospitalar, mas não com hiperóxia nas primeiras 24 horas na UTI e mortalidade em pacientes ventilados. ${ }^{15}$ Esses achados diferem dos estudos anteriores e sugerem que o impacto da hiperóxia precoce na mortalidade permanece incerto. 
Nosso estudo tem várias limitações. Primeiro, foi um estudo retrospectivo realizado em um único centro. Portanto, nossos resultados não podem ser generalizados e estudos prospectivos multicêntricos são necessários para confirmar esses achados. Em segundo lugar, o corte usado para hiperóxia $(>120$ $\mathrm{mmHg}$ ) foi selecionado com base na literatura atual. ${ }^{10,15,16} \mathrm{~A}$ $\mathrm{PaO}_{2}$ normal é definido pela British Thoracic Society entre 90 e $110 \mathrm{mmHg}$ para pacientes com menos de 70 anos de idade e de acordo com o nível do mar. ${ }^{21}$ No entanto, outros estudos usaram um corte diferente (isto é, $\mathrm{PaO}_{2} \geq 300$ $\mathrm{mmHg}$ ) para definir hiperoxemia. ${ }^{22-24}$ Em terceiro lugar, outra limitação deste estudo é que a condição clínica do paciente pode influenciar as decisões para mudar as configurações do ventilador e que poucas informações sobre a condição dos pacientes estiveram disponíveis para essa análise.

Apesar dessas limitações, o nosso estudo é o primeiro realizado em uma UTI brasileira, pesquisando a ocorrência de hiperóxia à admissão em pacientes sob ventilação mecânica. Esse estudo alerta para a necessidade de gerenciamento da oferta segura de oxigênio para esses pacientes e implementação de mudanças na prática diária.

\section{REFERÊNCIAS}

1. Suzuki S, Eastwood GM, Peck L, Glassford NJ, Bellomo R. Current oxygen management in mechanically ventilated patients: a prospective observational cohort study. J Crit Care. 2013;28:647-54.

2. Suzuki S, Eastwood GM, Glassford NJ, Peck L, Young H, GarciaAlvarez M, et al. Conservative oxygen therapy in mechanically ventilated patients: a pilot before-and-after trial. Crit Care Med. 2014;42:1414-22.

3. Rachmale S, Li G, Wilson G, Malinchoc M, Gajic O. Practice of excessive FIO2 and effect on pulmonary outcomes in mechanically ventilated patients with acute lung injury. Respir Care. 2012;57:1887-93.

4. Helmerhorst HJ, Schultz MJ, van der Voort PH, Bosman RJ, Juffermans NP, de Wilde RB, et al. Effectiveness and clinical outcomes of a two-step implementation of conservative oxygenation targets in critically ill patients: a before and after trial. Crit Care Med. 2016;44(3):554-63.

5. Panwar R, Hardie M, Bellomo R, Barrot L, Eastwood GM, Young PJ, et al. Conservative versus liberal oxygenation targets for mechanically ventilated patients, a pilot multicenter randomized controlled trial. Am J Respir Crit Care Med. 2016;193:43-51

6. Helmerhorst HJ, Roos-Blom MJ, van Westerloo DJ, de Jonge E. Association between arterial hyperoxia and outcome in subsets of critical illness: a systematic review, meta-analysis, and metaregression of cohort studies. Crit Care. 2015;43(7):1508-19.

7. Six S, Jaffal K, Ledoux G, Jaillette E, Wallet F, Nseir S. Hyperoxemia as a risk factor for ventilator-associated pneumonia. Crit Care. 2016;20:195.

8. Cornet AD, Kooter AJ, Peters MJ, Smulders YM. The potential harm of oxygen therapy in medical emergencies. Crit Care. 2013; $17: 313$.

9. Farquhar H, Weatherall M, Wijesinghe M, Perrin K, Ranchord A,
Serve também de fundamento para estudos sobre educação e orientação de todos os profissionais de saúde relacionados ao gerenciamento de oxigênio, incluindo médicos, residentes, enfermeiros e fisioterapeutas. O uso de lembretes periódicos de natureza eletrônica e/ou manual, bem como ferramentas de suporte à decisão para o grau de titulação de oxigênio, podem ser propostos, baseado em nossos resultados.

\section{CONCLUSÃO}

O percentual de hiperóxia à admissão na UTI em pacientes sob ventilação mecânica é elevado, e esse não deve ser negligenciado, devendo fazer parte da avaliação clínica diária. Mais estudos são necessários para estabelecer níveis de segurança quanto a oferta de oxigênio em subgrupos de pacientes críticos. Além disso, é importante o desenvolvimento de estratégias para gerenciamento adequado da oferta de oxigênio e a modificação da cultura do "mais é melhor" de suplementação de oxigênio no ambiente de terapia intensiva, ressaltando a importância do "menos é mais".

Simmonds M, Beasley R. Systematic review of studies of the effect of hyperoxia on coronary blood flow. Am Heart J. 2009;158:371-7.

10. de Jonge E, Peelen L, Keijzers PJ, Joore H, de Lange D, van der Voort PH, et al. Association between administered oxygen, arterial partial oxygen pressure and mortality in mechanically ventilated intensive care unit patients. Crit Care. 2008;12(6):R156.

11. Altemeier WA, Sinclair SE. Hyperoxia in the intensive care unit: why more is not always better. Curr Opin Crit Care. 2007;13:73-8.

12. Kavanagh BP. Goals and concerns for oxygenation in acute respiratory distress syndrome. Curr Opin Crit Care. 1998;4:16-20.

13. Knaus WA, Draper EA, Wagner DP, Zimmerman JE. APACHE II: a severity of disease classification system. Crit Care Med. 1985;13(10):818-29.

14. Vincent JL, Moreno R, Takala J, Willatts S, De Mendonça A, Bruining H, et al. The SOFA (Sepsis-related Organ Failure Assessment) score to describe organ dysfunction/failure. On behalf of the Working Group on Sepsis-Related Problems of the European Society of Intensive Care Medicine. Intensive Care Med. 1996;22(7):707-10.

15. Eastwood G, Bellomo R, Bailey M, Taori G, Pilcher D, Young P, et al. Arterial oxygen tension and mortality in mechanically ventilated patients. Intensive Care Med 2012;38(1):91-8.

16. Itagaki T, Nakano Y, Okuda N, Izawa M, Onodera M, Imanaka H, et al. Hyperoxemia in mechanically ventilated, critically ill subjects: incidence and related factors. Respir Care. 2015;60:335-40.

17. De Graaff AE, Dongelmans DA, Binnekade JM, de Jonge E. Clinicians' response to hyperoxia ventilated patients in a Dutch ICU depends on the level of FiO2. Intensive Care Med 2011;37:4651.

18. Patel VS, Sitapara RA, Gore A, Phan B, Sharma L, Sampat V, 
et al. High Mobility Group Box-1 mediates hyperoxia-induced impairment of Pseudomonas aeruginosa clearance and inflammatory lung injury in mice. Am J Respir Cell Mol Biol. 2013;48(3):280-7.

19. Kennedy TP, Nelson S. Hyperoxia, HMGB1, and ventilatorassociated pneumonia: reducing risk by practicing what we teach. Am J Respir Cell Mol Biol. 2013;48(3):269-70.

20. Greif R, Akca O, Horn EP, Kurz A, Sessler DI; Outcomes Research Group. Supplemental perioperative oxygen to reduce the incidence of surgical-wound infection. N Engl J Med. 2000;342(3):161-7.

21. O'Driscoll BR, Howard LS, Bucknall C, Welham SA, Davison AG. British Thoracic Society emergency oxygen audits. Thorax. 2011;66:734-5.
22. Kilgannon JH, Jones AE, Shapiro NI, Angelos MG, Milcarek B, Hunter $\mathrm{K}$, et al. Association between arterial hyperoxia following resuscitation from cardiac arrest and in-hospital mortality. JAMA. 2010;303:2165-71.

23. Bellomo R, Bailey M, Eastwood GM, Nichol A, Pilcher D, Hart GK, et al. Arterial hyperoxia and in-hospital mortality after resuscitation from cardiac arrest. Crit Care. 2011;15:R90.

24. Rincon F, Kang J, Maltenfort M, Vibbert M, Urtecho J, Athar MK, et al. Association between hyperoxia and mortality after stroke: a multicenter cohort study. Crit Care Med. 2014;42:387-96.

\section{Como citar:}

Aguiar LS, Beltrão BA, Aragão NL, Araújo VN, Viana AB Júnior, Gomes JG, et al. Ocorrência de hiperóxia em unidade de terapia intensiva adulto de um hospital universitário do nordeste do Brasil. Rev Med UFC. 2019 jul-set;59(3):6-10. 